[Technical Paper]

\title{
An Influence of the Module Structure on Reliability of Crystalline Silicon Solar Cells
}

\author{
Taeko Semba*, Genki Saito*, Shuichi Asao**, Katsuhiko Shirasawa**, and Hidetaka Takato** \\ *NAMICS Corporation, 3993 Nigorikawa, Niigata 950-3131, Japan \\ **Renewable Energy Research Center, National Institute of Advanced Industrial Science and Technology (AIST), 2-2-9 Machiikedai, Koriyama 963- \\ 0298, Japan
}

(Received August 23, 2019; accepted January 7, 2020, published April 1, 2020)

\begin{abstract}
To investigate the influence of the difference in module structures on the degradation of crystalline silicon solar cells, two different photovoltaic modules were fabricated, and a high-temperature and high-humidity test was carried out. The degradation modes of these modules were compared to each other using electrical characteristics and electroluminescence images. Degradation of outputs occurred in both modules, and different degradation modes were confirmed by electroluminescence images. The difference in degradation modes between these modular structures could be due to the difference in concentration distribution attributable to the moisture and acetic acid generated from the encapsulant inside the module.
\end{abstract}

Keywords: Aluminum, Corrosion, Damp Heat Test, Metallization, Moisture Ingress, Photovoltaic Module, Silver Paste

\section{Introduction}

With the growing interest in energy issues in recent years, the applications and importance of solar cells are increasing. To increase the lifetime power generation of solar cells, research is being carried out on high-power output structure and long-term reliability in modules as well as on high-conversion efficiency of cells by the development of design and materials. A conventional solar cell has a light-receiving surface (front side) and the backside, which does not receive light. As the front absorbs sunlight to generate electricity, the area occupied by metallization should preferably be small. For this reason, the front side metallization consists of fine lines called fingers for collecting current and a connecting portion with interconnectors called bus bars. Furthermore, it has a design called an $\mathrm{H}$ pattern where bus bars and fingers intersect vertically (Fig. 1.). The back-surface side of the cell is coated with $\mathrm{Al}$ paste on the entire surface and therefore the light cannot penetrate. Conventional solar cells are used with singlesided light-receiving type modules laminated with tempered glass on the front side and an opaque film, called back sheet on the backside. Conversely, metallization of the double-sided light-receiving solar cell is formed in an

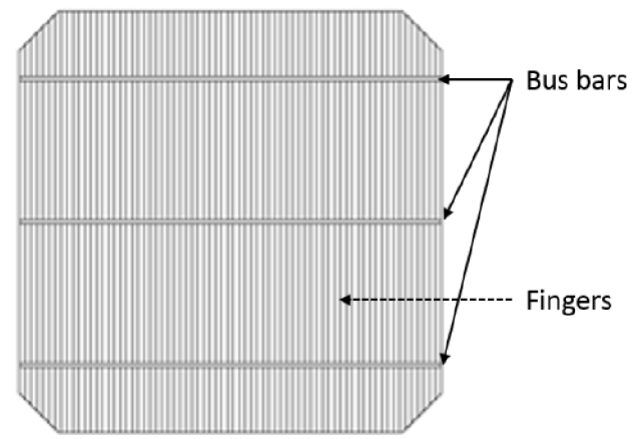

Fig. 1 Metallization screen printing design called $\mathrm{H}$ pattern of crystalline silicon solar cell. Three bold lines running in the horizontal direction are bus bars, thin lines running in the vertical direction are fingers.

$\mathrm{H}$ pattern on both the front and the back surface. As both surfaces of the solar cell are light-receiving they generate more power than a single-sided light-receiving surface (Fig. 2.).[1] To implement this concept, a double-sided glass structure is adopted in a module using a doublesided light-receiving cell. The photovoltaic module is designed with various structures.

Many reports on module reliability using single-sided light-receiving cells such as aluminum back surface field type passivated emitter and rear cell type are available. 
One of the causes of module output reduction is corrosion of the front side metallization because acetic acid generated by hydrolysis of ethylene vinyl-acetate copolymer used as an encapsulant corrodes the glass, which is one of the components of metallization.[2-5] This degradation is exacerbated by moisture entering from the back sheet, which is composed of polyvinyl fluoride (PVF), polyethylene terephthalate (PET), and PVF triple layer on the backside of the module. However, there are few reports on the influence of the structure of the double-sided light-receiving type modules on the reliability of the solar cell. The double-sided light receiving module has a longer lifetime than the single-sided light-receiving module, and it has been confirmed that the degradation mode is different.[6]

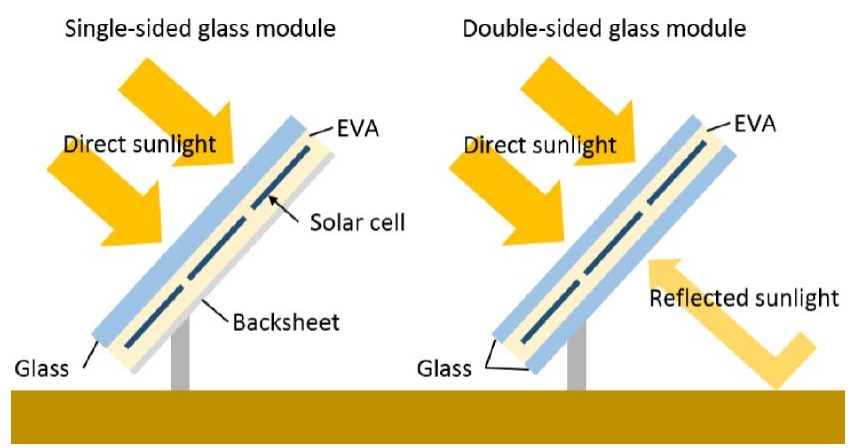

Fig. 2 Light penetration path of single-sided (left) and double-sided (right) glass module.
Glass is also used on the backside in the double-sided light receiving module, and it is assumed that no moisture permeation emerges from the backside.

We investigated how module structural differences affect cell degradation in a high-temperature and highhumidity test. Two types of module structures were fabricated using similar double-sided light-receiving solar cells called bifacial solar cells; one was a single-sided glass and the other was a double-sided module. We conducted hightemperature and high-humidity tests using these modules. A comparison of the electrical characteristics and electroluminescence (EL) images of these modules after the test confirmed that the degradation mode differed depending on the structure of the module. These results showed that the relationship between moisture intrusion and acetic acid concentration changes depending on the module structure, and this affects cell degradation.

\section{Experiment}

\subsection{Sample preparation}

Figure 3 shows the process flow of the sample preparation. The bifacial solar cells were n-type monocrystalline silicon of $157.5 \times 157.5 \mathrm{~mm}^{2}$ pseudo square, and the $\mathrm{p}^{+}$ emitter layer was formed on the front surface side while the $\mathrm{n}^{+}$back surface field (BSF) layer was formed on the opposite side (Fig. 3(1)). The surface of the solar cell has

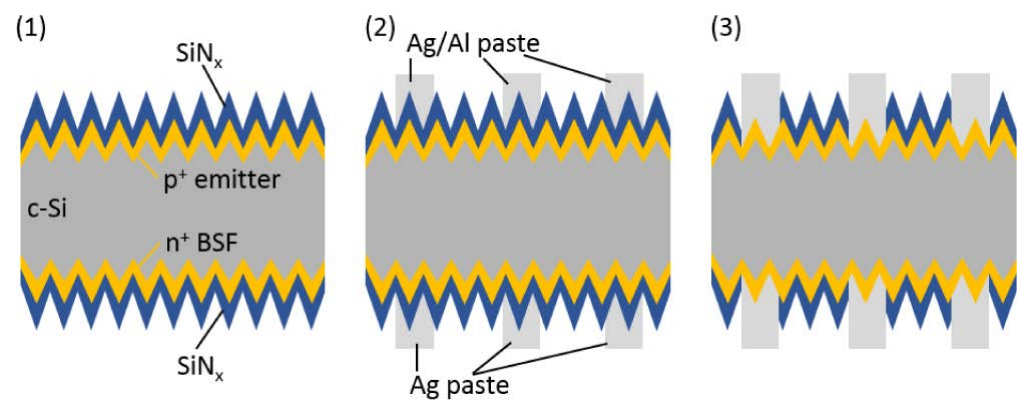

(4)

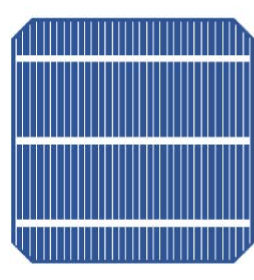

(5)

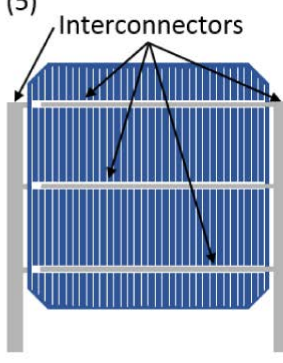

(6)

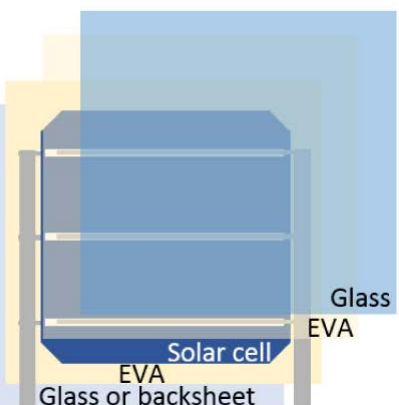

Fig. 3 Process flow of a sample preparation: (1) Structuring a bifacial Si solar cell, (2) Screen printing metallization paste on both sides, (3) Firing to enable the fire-through to form contact between the emitter and metallization, (4) Forming a bifacial surface cell (5) Soldering to connect interconnectors, and (6) Laminating with Glass, EVA, and/or backsheet. 
Table 1 Appearance image and schematic design of samples.

\begin{tabular}{c|c|c}
\hline $\begin{array}{c}\text { Module } \\
\text { structure }\end{array}$ & $\begin{array}{c}\text { Appearance } \\
\text { image }\end{array}$ & $\begin{array}{c}\text { Schematic } \\
\text { cross-sectional view }\end{array}$ \\
\hline $\begin{array}{c}\text { Glass backsheet } \\
\text { (G/BS) module }\end{array}$ & $\vdots$ & Glass \\
& 1 & EVA \\
\hline $\begin{array}{c}\text { Double-sided } \\
\text { glass (G/G) }\end{array}$ & Solar cell \\
module & EVA \\
& Backsheet \\
\hline
\end{tabular}

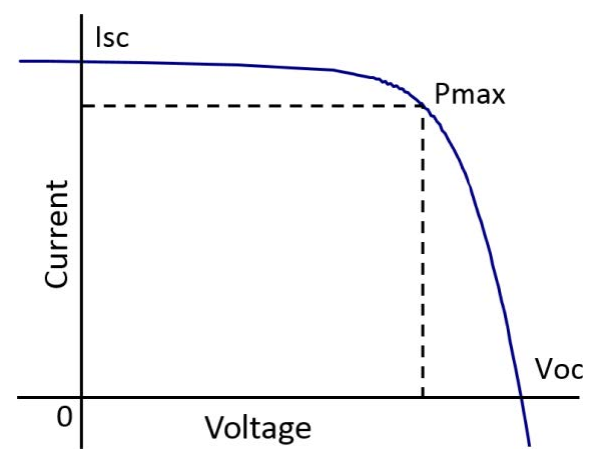

Fig. 4 An example of an I-V curve of a PV module at 1 sun irradiation.

Table 2 Electrical characteristics and rate of change of modules.

\begin{tabular}{c|c|c|c|c|c|c|c|c|c}
\hline \multirow{2}{*}{ Time } & \multirow{2}{*}{ Structure } & \multicolumn{4}{|c|}{ Electrical Characteristics } & \multicolumn{4}{c}{ Rate of Change } \\
\cline { 3 - 12 } & & Isc (A) & Voc (V) & Pmax (W) & FF (\%) & Isc (\%) & Voc (\%) & Pmax (\%) & FF (\%) \\
\hline \multirow{2}{*}{$0 \mathrm{~h}$} & G/BS & 9.412 & 0.654 & 4.376 & 71.1 & & & & \\
\cline { 2 - 12 } & G/G & 9.176 & 0.653 & 4.318 & 72.0 & & & & \\
\hline \multirow{2}{*}{$1,464 \mathrm{~h}$} & G/BS & 4.077 & 0.646 & 0.817 & 31.0 & -56.7 & -1.2 & -81.3 & -56.4 \\
\cline { 2 - 12 } & G/G & 7.097 & 0.650 & 1.388 & 30.1 & -22.7 & -0.5 & -67.9 & -58.3 \\
\hline
\end{tabular}

an uneven structure: the texture, which reduces the reflection loss due to multiple reflections of light and has the effect of confining the infiltrated light inside the solar cell. On the surface of the cell, a $\mathrm{SiN}_{\mathrm{x}}$ layer was deposited as a passivation layer. $\mathrm{An} \mathrm{Ag} / \mathrm{Al}$ paste was applied on the $\mathrm{p}^{+}$ emitter side and an Ag paste was applied on the $n^{+}$BSF side by screen-printing on each surface (Fig. 3(2)). The $\mathrm{Ag} / \mathrm{Al}$ paste used for the $\mathrm{p}^{+}$emitter on the front side was made of $\mathrm{Ag}$, glass frit, resin, solvent, and $\mathrm{Al}$ (added to obtain good contact between metallization and the $\mathrm{p}^{+}$emitter layer). Conversely, $\mathrm{Al}$ was not added to the $\mathrm{Ag}$ paste on the $\mathrm{n}^{+} \mathrm{BSF}$ side on the backside. The firing was carried out at $820^{\circ} \mathrm{C}$ in a commercial belt furnace for the duration of approximately $40 \mathrm{~s}$. During firing, the resin in the paste burned out; the glass frit melted and a liquid phase sintering of $\mathrm{Ag}$ was obtained. At high temperature, the melted glass reached the interface of the silicon substrate, and the $\mathrm{SiN}_{\mathrm{x}}$ layer was removed by a chemical reaction with melted glass called fire-through. The insulating $\mathrm{SiN}_{\mathrm{x}}$ layer was removed, and metallization and the $\mathrm{p}^{+}$emitter (or the $\mathrm{n}^{+}$BSF) layer connected electrically, formed an electron extraction path (Fig. 3(3)). After firing, both sides of the bifacial solar cell looked like Fig. 3(4). Thereafter, in the cooling process, the glass solidified between the Si substrate and Ag, providing good adhesion by the anchor effect. After connecting the interconnectors to the bus bars of the solar cell (Fig. 3(5)), a double-sided glass $(\mathrm{G} / \mathrm{G})$ module and a glass back sheet (G/BS) module were laminated with ethylene vinyl-acetate copolymer sheet as an encapsulant (Fig. 3(6)). Table 1 shows the appearance of the photovoltaic module fabricated and schematic cross-sectional view of the structure. High temperature and high humidity test were conducted using these modules.

\subsection{High temperature and high humidity test}

The high temperature and high humidity test were conducted under the conditions of $95{ }^{\circ} \mathrm{C} / 95 \% \mathrm{RH}$. When the test time reached $1,464 \mathrm{~h}$, the electrical characteristics of the modules were measured by a solar simulator, and the rate of change from the initial state was calculated for short circuit current (Isc), open-circuit voltage (Voc), maximum power point (Pmax), and fill factor (FF) at 1 sun irradiation. Figure 4 shows an IV curve of the output characteristics of the PV module during power generation. Pmax is the point where the product of voltage and current is maximum on the IV curve. FF is expressed by

$$
F F=\frac{P \max }{I s c * V o c} .
$$

The degraded part in the modules was investigated by checking the increase in series resistance using EL inspection and comparing the states before and after the test.

\section{Results and Discussion}

\subsection{Electrical characteristics}

Table 2 lists the electrical characteristics of the G/BS and $\mathrm{G} / \mathrm{G}$ modules at $0 \mathrm{~h}$ and 1,464 $\mathrm{h}$ at the test time and 
change rate from the initial state. The FF of the G/BS module decreased from $71.1 \%$ to $31.0 \%$, and the Isc also decreased significantly from $9.412 \mathrm{~A}$ to $4.077 \mathrm{~A}$. The rate of change was $-56.4 \%$ for $\mathrm{FF}$ and $-56.7 \%$ for Isc and the output was $0.817 \mathrm{~W}$, which is a downward decrease by $-81.3 \%$ with little power generation. Conversely, the FF of the G/G module decreased from $72.0 \%$ to $30.1 \%$ and the rate of change also became $-58.3 \%$, which is the same as the G/ BS module, but the Isc decreased from $9.176 \mathrm{~A}$ to $7.097 \mathrm{~A}$, and the rate of change of became $-22.7 \%$, which is smaller than the G/BS module. Therefore, the Pmax of the G/G module was $1.388 \mathrm{~W}$, and this was higher than the G/BS module. In both modules, the rate of decrease in the Voc was small, and there was almost no drop in the output due to the degradation of the $\mathrm{Si}$ substrate. The degradation rate of the electrical characteristics observed in this test was faster than that observed in ordinary crystalline silicon solar module. The reason for these results is that the paste used is a modified composition and the test conditions of $95{ }^{\circ} \mathrm{C} / 95 \% \mathrm{RH}$ are more unfavorable than the general $85{ }^{\circ} \mathrm{C} / 85 \% \mathrm{RH}$ conditions. However, under the standard and test conditions conducted in this study, the degradation tendency of the electrical characteristics in the module with the G/BS module structure using the conventional single-sided light-receiving cell shows the same mode. The rate of degradation in time has increased because the acceleration of degradation became large.

\subsection{Electroluminescence inspection}

Next, the EL inspection of each module was carried out and the defective part and its degradation mode were confirmed. EL inspection analyzes an image by photographing electrons emitted from the electrons and holes recombined in the semiconductor by applying an electric field to the solar cell. When an electric field is applied to the cell, the portion through which electricity flows emits electrons, but the portion where electricity does not flow remains dark. Therefore, the defective part is shown as a dark area in the image, which indicates failure. This method has a merit to find a variety of failures, such as cracks of a cell, which cannot be detected from the appearance but can lead to a decrease in the power generation; disconnection/connection failure of the interconnector; or increase in resistance between the finger metallization and emitter of the Si substrate. After the test, the dark part in the EL spreads around the cell edge, and a dark spot exists in the plaque in the central part in the G/BS module (Fig. 5). Conversely, the bright and dark part exist like a mosaic on the whole cell surface in the G/G module (Fig. 6). This

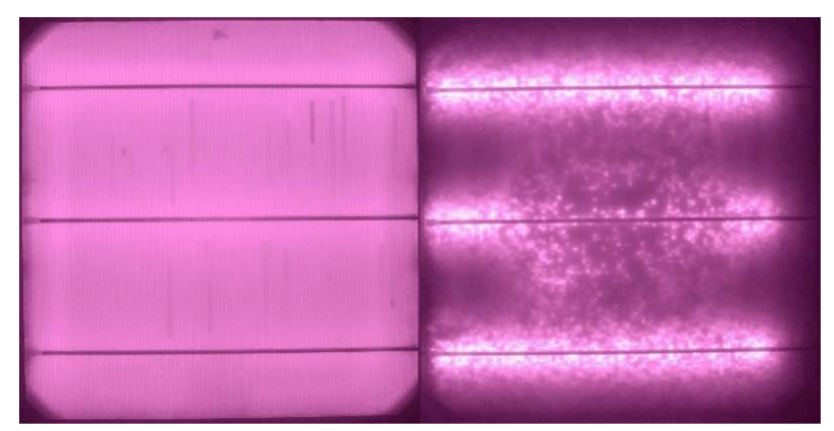

Fig. 5 EL image of the front side of the G/BS module before (left) and after (right) the test.

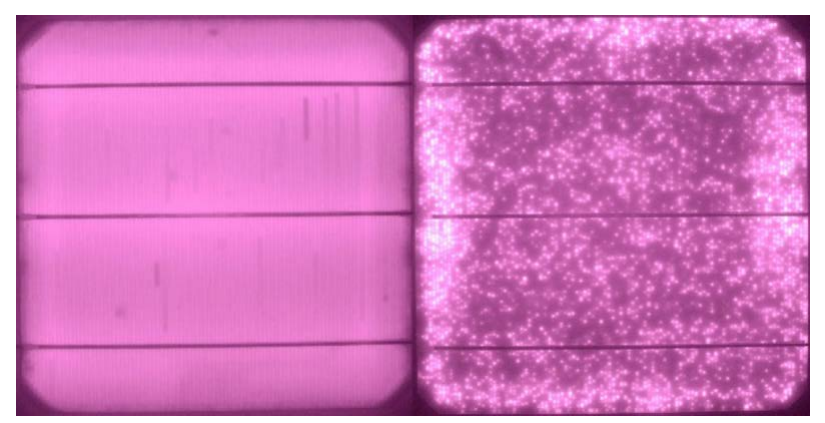

Fig. 6 EL image of the front side of the G/G module before (left) and after (right) the test.

mosaic-like EL pattern was given similar pictures when EL inspection was performed from both sides of the $G / G$ module. We did not find the spread of the dark area around the cell edge with the G/BS module. From these facts, it was found that the occurrence of the EL dark part, i.e., the degradation mode, differs depending on the module structure even when using the same solar cell.

\section{Discussion of the Degradation Mechanism}

Generally, the degradation of PV modules is caused by an increase in the series resistance of solar cells owing to high temperature and high humidity test. The first step in the effect of increasing the series resistance is to reduce the FF due to a decrease in Pmax. The main causes of the series resistance of the solar cells are an increase in contact resistance between the emitter layers due to corrosion of the finger metallization, a connection failure of the bus bar, and the interconnector ribbon due to peeling. If a connection failure occurs between the bus bar and the interconnector, EL dark area will appear in a wide area centering on the bus bar. The area around the bus bar of the module was bright after the test in EL image, and the EL dark area was spread at the center and edge of the cell with finger metallization. In other words, the decrease in output is caused by an increase in contact resistance between the finger metallization and emitter layer. In the 
next step of increasing resistance, connection failure occurs between the emitter and finger metallization. Due to light reception, it becomes difficult to distinguish the current generated inside the solar cell, thereby causing a decrease in the Isc.

The corrosion of the front side metallization of the crystalline silicon photovoltaic module in the test is closely related to the moisture that penetrates the module and acetic acid generated by hydrolysis of ethylene-vinyl acetate copolymer as the encapsulant. Kempe and SculatiMeillaud conducted a finite-element method (FEM) analysis on moisture penetration of the module and found that the amount and distribution of moisture in the module differed depending on the module structure.[6, 7] Moisture enters the inside of the module through the back sheet used in the G/BS module, permeates from the backside to the peripheral part, and reaches the cell surface. Therefore, the edge of the cell light-receiving surface has more moisture than the center of the cell. Degradation from the edge portion of the solar cell in the G/BS module has also been widely confirmed in the high temperature and high humidity test of a conventional glass back sheet type module using a general single-sided light-receiving type cell. This degradation is affected by moisture penetration inside the module. In the center of the cell where the mosaic-like EL dark area occurs, it takes time for moisture to reach and saturate, and so the influence of moisture is considerably smaller than at the cell edge. In the $G / G$ module, moisture intrudes from the ethylene vinyl-acetate on the side of the module edge. The time taken for the moisture to saturate the module is longer than it takes in the G/BS module, and the degradation of the electrical characteristics is slow. The degradation mode seen from the EL image of the $\mathrm{G} / \mathrm{G}$ module has a mosaic-like EL dark area across the cell surface, which is identical to that seen in the center of the G/BS module. Moisture inside the G/G module could be on the same level as at the center of the G/BS module.

The degradation of the output of the PV module is caused by the presence of acetic acid. Masuda et al. quantified the amount of acetic acid generated in the module using ion chromatography. [8] The results confirmed that the amount of acetic acid in the G/BS module increased from the periphery of the cell immediately after the start of the high temperature and high humidity test and started to increase after a certain period of time in the center of the cell. Similar results were obtained in the study using Sn by Hamaoka et al.[9] The amount of acetic acid in the
G/G module was not quantified, and hence cannot be compared due to differences in the structure. However, because the rate of decrease in the Pmax is smaller in the $\mathrm{G} / \mathrm{G}$ module than in the G/BS module, the amount of acetic acid generation of $G / G$ is expected to be smaller than the one in the G/BS module.

Due to the relationship between the moisture penetration and amount of acetic acid, the EL dark area of the cell edge of the G/BS module occurs in the area where a large amount of moisture and acetic acid exist from the beginning of the high temperature and high humidity test. Conversely, in the EL dark areas in the mosaic-like pattern, we saw the area where the amount of moisture is small and the acetic acid concentration increases significantly from the middle of the test. This suggests that the two degradation modes observed in EL images are different metallization corrosion phenomena. Although the amount of acetic acid inside the $\mathrm{G} / \mathrm{G}$ module is not known, the occurrence of the EL dark pattern of the mosaic suggests that it resembles the state of the central part of the G/BS module.

\section{Summary}

We investigated the influence of the structure of the module on the degradation of the solar cell in the hightemperature high-humidity test. The tests were carried out using two types of solar modules, G/BS and G/G, and the electrical characteristics before and after the test were compared with the degradation mode in EL images. After the test that lasted 1,464 h, the electrical characteristics of the module were significantly reduced, especially the G/ BS module rate of decline which was large. The EL dark part spread from the edge of the solar cell in the module in the G/BS module. This is the same degradation mode as used in other previous studies, which is caused by the acetic acid generated within the module and the penetration of moisture into the module. In addition, in the central part of the $\mathrm{G} / \mathrm{BS}$ module and the $\mathrm{G} / \mathrm{G}$ module, the EL dark spots occurring as a mosaic-like degradation were observed. It was suggested that this degradation occurred in the module with a particularly high concentration of acetic acid. From the above analysis, it was found that not only the change in electrical characteristics but also the mechanism of failure occurrence varies depending on the module structure.

\section{References}

[1] Sanyo Energy Corporation, "Bifacial Photovoltaic 
Module HIT Double 195 Photovoltaic Module,” 2008.

[2] C. Peike, S. Hoffmann, P. Hülsmann, B. Thaidigsmann, K. A. Wei $\beta$, M. Koehl, and P. Bentz, "Origin of Dampheat Induced Cell Degradation,” Solar Energy Materials \& Solar Cells, Vol. 116, pp. 49-54, 2013.

[3] A. Kraft, L. Labusch, T. Ensslen, I. Dürr, J. Bartsch, M. Glatthaar, S. Glunz, and H. Reinecke, "Investigation of Acetic Acid Corrosion Impact on Printed Solar Cell Contacts," IEEE Journal of Photovoltaics, Vol. 5, No. 3, pp. 736-743, 2015.

[4] T. Tanahashi, N. Sakamoto, H. Shibata, and A. Masuda, "Electrical Detection of Gap Formation underneath Finger Electrodes on c-Si PV Cells Exposed to Acetic Acid Vapor under Hydrothermal Conditions," Proc. 43rd IEEE Photovoltaic Specialists Conference (PVSC), pp. 1075-1079, 2016.

[5] T. Semba, T. Shimada, K. Yamada, K. Shirasawa, and H. Takato, "Corrosion of the Glass and Formation of Lead Compounds in the Metallization by High Temperature and High Humidity Test of Crystalline Silicon PV Module," Proc. 7th World Conference on Pho- tovoltaic Energy Conversion (WCPEC-7), pp. 13331335, 2018.

[6] F. Sculati-Meillaud, "Reliability of PV modules and long-term performance prediction,” Swiss Photonics Workshop, 2015.

[7] M. D. Kempe, "Modeling of rates of moisture ingress into photovoltaic modules," Solar Energy Materials and Solar Cells, Vol. 90, pp. 2720-2738, 2006.

[8] A. Masuda, N. Uchiyama, and Y. Hara, "Degradation by acetic acid for crystalline Si photovoltaic modules," Japan Journal of Applied Physics, Vol. 54, 04DR04, 2015.

[9] R. Hamaoka, K. Iwami, T. Itayama, H. Nagasaki, S. Takemoto, C. Yamamoto, Y. Hara, A. Masuda, and N. Umeda, "Detection of acetic acid produced in photovoltaic modules based on tin film corrosion during damp heat test," Japan Journal of Applied Physics, Vol. 57, 08RG16, 2018.

Taeko Semba 